\title{
EXPLORING THE MOBILE DEVICES AS A NEW FRONTIER FOR THE E-LEARNING IN INSTRUMENTATION AND MEASUREMENT
}

\author{
Drago Cmuk ${ }^{1)}$, Domenico Grimaldi ${ }^{2)}$, Sergio Rapuano ${ }^{3)}$ \\ ${ }^{1)}$ Faculty of Electrical Engineering and Computing, Zagreb, Croatia, \\ ${ }^{2)}$ DEIS, University of Calabria, 87036 Rende (CS), Italy, \\ ${ }^{3)}$ Dept. of Engineering, University of Sannio, Corso Garibaldi 107, 82100 Benevento, Italy, \\ E-mail:drago.cmuk@unisannio.it, grimaldi@deis.unical.it, rapuano@unisannio.it
}

\begin{abstract}
The mobile-learning establishes a new frontier for the research of the e-learning in the electrical and electronic measurement and instrumentation. Indeed, the reduced functionalities of the mobile devices must be integrated with the typologies of the services delivered to the student. By referring to the Remote Didactic Laboratory Laboratorio Didattico Remoto - LA.DI.RE. "G. Savastano" the delivered services taken into account are: experiment visualization, experiment control, and experiment creation. In order to enable the user of the mobile device to view, control and create the experiment application, several software architectures have been considered. Finally, the description of the solution adopted is given in the framework of the LA.DI.RE. "G. Savastano".
\end{abstract}

Keywords: m-learning, e-learning, virtual laboratory, mobile device.

\section{INTRODUCTION}

The e-learning took learning away from the classroom or campus, the mobile-learning is taking learning away anytime and anywhere. While elearning is an alternative to classroom learning, $\mathrm{m}$ learning is the complementary activity to both elearning and traditional learning [1]. M-learning respects that the user would like to interact with educational resources whilst away from their normal place of learning-classroom or computer. It is gaining appeal among younger generations who have grown up using portable video game devices and wireless technology. In this sense, m-learning appeals not only to those who need learning portable, but to those who have grown up with a cognitive disposition towards using mobile devices whether or not they have the need for true portability in their learning.

The m-learning has been recognized by the European Union Commission which founded the m-learning project [2].

The m-learning has been around for longer than elearning, with the paperback book and other portable resources, but technology is what shapes today's the usage. Technology now allows carrying vast resources in the pockets and accessing these wherever we find convenient. While the opportunities that m-learning devices present are news, the challenges are quite old as a consequence of the smaller screen sizes, the limited processing power, and the reduced input capabilities. These challenges mean that adapting existing e-learning services and content to $\mathrm{m}$-learning is not a trivial task.

Goal of the author is not to introduce m-learning as a novel and popular way of learning that can substitute the older, unpopular types of learning, but it should be considered rather as an important part of Blended learning. With today's prevalence of high technology in many countries, blended learning often refers specifically to the use of resources which combine e-learning or mlearning with classical (classroom courses etc.) educational resources. In the paper some aspects of the mlearning are address in the field of the education in electrical and electronic measurement and instrumentation. This engineering field caries out certain particularities which will be solved in practical way, so that optimized blended learning process in electrical and electronic measurement and instrumentation curriculum becomes realizable to the highest degree possible.

Indeed, practical experience is a crucial step in effective transfer of knowledge to students in electrical engineering education. But necessity for experimentation is surpassed by common problems in creating and maintaining laboratory work in undergraduate curriculum [3].

In [4] is highlighted that the Remote Didactic Laboratory Laboratorio Didattico Remoto - LA.DI.RE. "G. Savastano" through complete Learning Management System (LMS) enables students to execute geographically 
distributed experiments on the real equipment based on a thin client approach. Following the newest approaches to the e-learning optimization and extension, the LA.DI.RE. "G. Savastano" includes testing of the effectiveness, implementation of the newest standards, realization of feedback aided multilanguage experiments, adaptation of LMS to the execution of experiments.

In the work of extending the functionalities of the LA.DI.RE. "G. Savastano" to mobile devices, the primal goal is to create the software platform which will allow students to visualize, control and create experiments. This last aspect makes the major difference from the common m-learning systems.

In the following, before to present the technical solutions adopted for implement the m-learning, the aspects concerning the delivered services of the LA.DI.RE are discussed. These aspects are fundamentals to design the proper architecture realizing the m-learning. Successively, the technical characteristics of the mobile devices giving direction to the research are summarized. Finally, the software architectures to introduce the mlearning as a new feature of the LA.DI.RE. "G. Savastano" are explored.

\section{DELIVERED SERVICES OF LA.DI.RE. "G. SAVASTANO"}

The Remote Didactic Laboratory LA.DI.RE. "G. Savastano" is a virtual learning environment devoted to the teaching of electric and electronic measurement that integrates an off-the-shelf LMS and a geographically distributed laboratory, accessible from the Web by using a simple browser. The distributed laboratory is accessed through the LMS executed on a central server that delivers such functionality to users by means of Web Services technologies [5] and the Virtual Instruments (VIs), controlling the instrumentation.

This platform delivers the typical functionalities of a common LMS, including user authentication, management and tracking of learning process at user level. Moreover, it provides several innovative functionalities encapsulating in specific Learning Objects (LOs) the remote control of measurement instrumentation. This objective has been achieved by developing an additive module for the LMS Inform@ from Didagroup. The module ensures the integration of VIs written in LabVIEW in the LMS as LOs, thus enabling remote users to transparently get the control of a real measurement instrument and to display the measurement results within the normal learning activities.

The remote laboratory is distributed on geographical scale since the measurement instruments are physically located in laboratories belonging to different universities. At now, there are four laboratories involved, two of them have been realized and operate, at the University of Sannio in Benevento, and at the "Mediterranea" University of Reggio Calabria in Italy. Two more laboratories are going to be realized at the University of Zagreb (Croatia) and at the Technical University of Kosice (Slovak Republic). The Web address is:

www.misureremote.unisannio.it

The access to the measurement instruments is handled by a scheduling system which, transparently through specific scheduling policies, connects the user to a specific physical laboratory in which the required measurement instruments are available.

Different user's profiles are managed by the system: student, teacher and administrator.

The services delivered by the remote measurement laboratory module to the student are mainly the following:

- Synchronous virtual laboratory, this service allows the student to follow on-line a laboratory activity hold by the teacher. The student can see on his/her display the desktop of the server used by the teacher to control the measurement instruments involved in the experiment. The experiment is carried out by operating on the front panel of the LabVIEW VI controlling all the involved instrumentation. Of course, the students should be logged in the system during the scheduled lab session. The data stream from the physical laboratory to the students can be sent in multicast mode. No automated scheduling policy is foreseen for such kind of activity.

- Experiment visualization, this service allows the student to observe the automatic execution of the experiment to take practice with the experiment procedure. This kind of service can de delivered to the students at each time of the day and all the times they need it without supervision.

- Experiment Control, this service allows the student to perform an experiment controlling remotely one or more instruments and observing them by means of a camera. The student can choose a specific experiment in a set of predefined ones and he can run it only if the required measurement instruments are currently available.

- Experiment Creation, this service allows the student to create remotely an experiment interacting directly with specialized software executed on the servers used to control the measurement instruments. This feature enables the adoption of PBL as didactic model. Under the supervision of the teacher, the students can develop a specific project producing, in an individual or collaborative manner, the VI to control a set of real instruments.

The services delivered to the teacher are related to the 
remote handling of the available experiments (remote creation, modification and removal of experiments, etc.).

Finally, the administrator is responsible of the correct working of the overall distributed system and of handling of the user profiles.

Only the last three asynchronous services are taken into account for the m-learning: experiment visualization, experiment control, and experiment creation.

\section{TECHNICAL CHARACTERISTICS OF THE MOBILE DEVICES}

The limitations of the technical characteristics of the mobile devices refining space of the possible solutions and giving directions to research and development of the software architecture are the following:

- Hardware limitations: biggest limits are the small displays and control-navigation problems for mobile devices without touch-screen. Reduced display size without any standardization requires adaptive environment, and special requirements on the Graphical User Interface (GUI) creation. On the other hand, navigation problems require particular solutions for experiments control and creation. Limited processor power is also a strong drawback because applications must be very "light", consuming memory as less as possible to reduce response time of the applications.

The wide variety of the mobile devices can be split in two categories. First one is represented by so-called smart phones, while other is represented by the Personal Digital Assistant (PDA). Crucial difference between the two groups is that usually smart phones do not have touch screen or any pointing devices, while the PDA usually has, which is an important issue. Also, PDA's have bigger memory resources and processor power. Similar separation is used for Java Mobile Edition (J2ME) configuration specifications [7]. It forced the development to flow in two different directions.

- Software limitations: mobile devices have wide variety of software version and producers which are sources of huge problems for developer. Starting from operating system, which has strict requirements for creating applications or Web based solutions, the supported programming languages differ greatly. Moreover, even the same operating systems (like Windows CE) through its versions support different levels of programming languages, or at least different levels of it, while the ubiquitous browser Internet Explorer Mobile (IE Mobile) or Opera Mobile supports limited set of JavaScript, Document Object Model and XML. Java ${ }^{\mathrm{TM}}$ Platform, Micro Edition (Java ME) which is one of the most ubiquitous application platform for mobile, varies greatly in versions and built-in packages from device to device. The same problem consist with the browser capabilities on supporting JavaScript and other web based languages.

- Low bandwidth: wireless LAN is not always available to the potential user, and for sure it does not fulfill "anytime and anywhere" requirements, so the system should be able to work over UMTS, EDGE or GPRS connections. The latter has high latency and restricted bandwidth which is a big drawback for applications or services using it.

\section{SOFTWARE ARCHITECTURE FOR THE M-LEARNING}

In the following is explored the possibility to use the mobile devices for the m-learning in electrical and electronic measurement and instrumentation by referring to the delivered services of the LA.DI.RE. "G. Savastano".

In particular, the functionalities of the mobile devices must integrate with the three asynchronous typologies of the services delivered to the student by the remote measurement laboratory: Experiment Visualization, Experiment Control, and Experiment Creation.

In order to enable the user to view and control the experiment application, several methods have been considered and tested. But, only limited number of them could be used in existing system. In the contrary, only one method is able to permit the user to create the experiment. These methods are described in the following.

\section{A. Experiment Visualization}

Remote experiment visualizations are normally a static GUI where only data presentation is dynamic (graphs, diagrams, numerical results etc.). Thus, every optimized solution will separate data from its presentation. In other words, it is necessary to reduce the traffic as possible to enable effective and meaningful data visualization of the experiment. This request reduces highly possible solutions because the separation of data and the visualization usually requires proprietary protocols, and require for the creator of the experiment to be able to write the experiment with certain level of understanding the communication protocols. This way becomes closed for a huge number of already developed experiments or hardware that can be controlled only by means of proprietary applications.

LabVIEW is one of todays most recognised and used programming tools for the development of the measurement software. Some of the best solutions are proprietary solutions of the $\mathrm{NI}^{\mathrm{TM}}$. The first one consists in realizing two VIs, one on the client side and another on the server side communicating via the TCP/IP protocol of the proprietary Datasocket technology. The second one relies on LabVIEW Web server that gives the possibility of viewing the image of VI running on the server 
machine, while for accessing and controlling it the client should have LabVIEW runtime environment installed.

Several solutions have also been proposed implementing a specific client-server couple using object oriented programming languages, instead of using LabVIEW. National Instruments already provides a LabView Web browser client and server-side software to connect to it. These modules, however, are better adapted for factory automation behind a firewall than the open Internet. This solution as specific client-server structures has the following advantages: (i) high efficiency from the bandwidth occupation point of view, (ii) light clients that don't require specific plug-ins, and (iii) high portability, when open technologies are used on the client side. Their main disadvantage is the limited reusability of already developed experiments and the necessity of developing new versions of existing VIs. In the proposed approach, those problems were successfully solved. Thin-client paradigm splits the presentation layer between client and server. The presentation logic runs on the server, while the thin client has the only task of showing the GUI that often reproduces window of the application running on the server. This approach gives the possibility of providing remote access to any application, written in whichever language, running locally on the server and requiring only an Internet browser on the client side.

Many implementations of the thin client paradigm have been proposed by researchers and deployed commercially. The thin-client platforms that have been evaluated are: Remote Desktop Protocol (RDP), Independent Computing Architecture (ICA), X-Window and Virtual Network Computing (VNC). Those platforms were evaluated in [7]. The RDP protocol was chosen, for its low cost respect to ICA, and higher efficiency respect to X-Window and VNC. RDP is a proprietary protocol of Microsoft, and allows direct connection to the Windows server. It supports different mechanisms to reduce the amount of transmitted data (compression, cashing of bitmaps in RAM), which is very important for applications that use a large amount of bitmaps. Thin client approach that was used for visualization of remote experiments in PC browsers has been used as a basis for delivering similar content to mobile devices.

Starting from ProperJava RDP client, a specific server-client structure has been realized using web services and Asynchronous JavaScript and XML: AJAX is the technique used in web application development. AJAX is a browser technology that involves the use of existing Web standards and technologies (XML/XHTML, DOM, CSS, JavaScript, XHR XMLHttpRequest) to create more responsive Web applications that reduce bandwidth usage by avoiding full page refreshes and providing a more "desktop application-like" user experience. Applications created by AJAX are generally classed in the category of Rich Internet Applications (RIA).
Current employed system is based on a modified ProperJavaRDP client which is used to connect to a server delivering experiment and running windows terminal services. It grabs the image of experiment application window and through XMLHTTPRequest it handles it dynamically to a "lightweight" web page which handles presentation. Since dimensions of the image are very small on mobile devices, some cashing algorithms would be just consuming processor power of the sever disabling it to be highly scalable, while handheld devices would loose their limited resources on cashing and compression, with small benefit in bandwidth usage.

\section{B. Experiment Control}

The control of the experiment is based on the java script which "catches" mouse coordinates and key pressed from the keyboard and transfer it to the particular web server which passes the data trough the ProperJavRDP client to the originating experiment allowing control. Web pages are also dynamically loading with JavaScripts, which reduces start-up time of experiment. Adaptive resolution of the monitoring web page which will enable optimal image size for big variety of devices is still under construction.

Possible alternatives have been tested in order to give the best performance. Java Mobile Edition has been abandoned because the VI that comes with Java Virtual Machine (JVM) is generally a kilobyte size Virtual Machine [7]. Therefore, it unable to handle demanding issues like image handling, presentation and socket communication with server. Sun abandoned development of usable JVM for PDA's, so currently exist only as a commercial product form IBM (Workplace Client Technology, Micro Edition). Other possible solution was to develop application in C\#. Lack of this solution was that it would work only on Microsoft Mobile platforms.

\section{EXPERIMENT CREATION}

The experiment creation by the mobile device is based on new design approach of the innovative VI proposed in [8]. This approach permits to overcome the difficulties arising from the reduced dimension of the memory of the PDA, and to the user the dynamical configuration of the measurement procedure by operating on the PDA. In this manner the user can customize the measurement procedure operating on the PDA, once download on demand the innovative VIs from the server. This approach is based on the organization of the innovative VI, named module, in both autonomous and self containing entities on the basis of the characteristics of the Measurement Instrument (MI). The communication among the modules is permitted by using a virtual bus implemented into the PDA. 


\section{DESIGN APPROACH OF THE MODULE FOR EXPERIMENT CREATION}

The traditional approach to design the VI is based on the implementation of all the functionalities of the MI. All the functionalities are available always by means of the GUI, and the user employs only those required by the measurement procedure. The result of this approach is the complete mapping of the MI into the VI (Fig.1a). Alternatively, only the functionalities of the MI required by the measurement procedure can be implemented by selecting the corresponding cluster of commands, as shown in Fig.1b. In this case the VI is created and customized on the basis of the complete measurement procedure. The selected functionalities are available always by means of the customized GUI of the measurement procedure. The advantage consists in the reduction of the memory size. The inconvenient consists in re-build the VI if new functionalities of the MI needs.
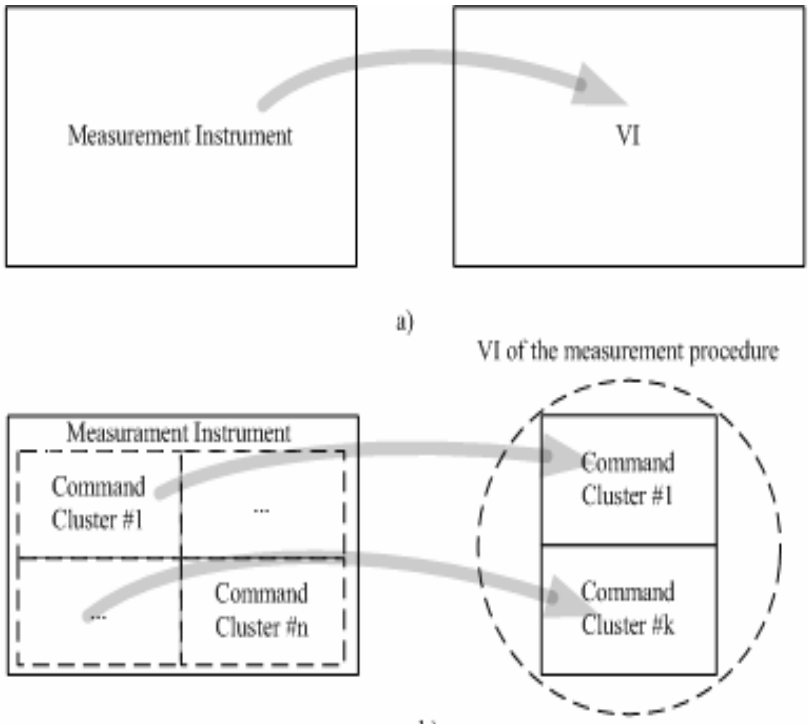

b)

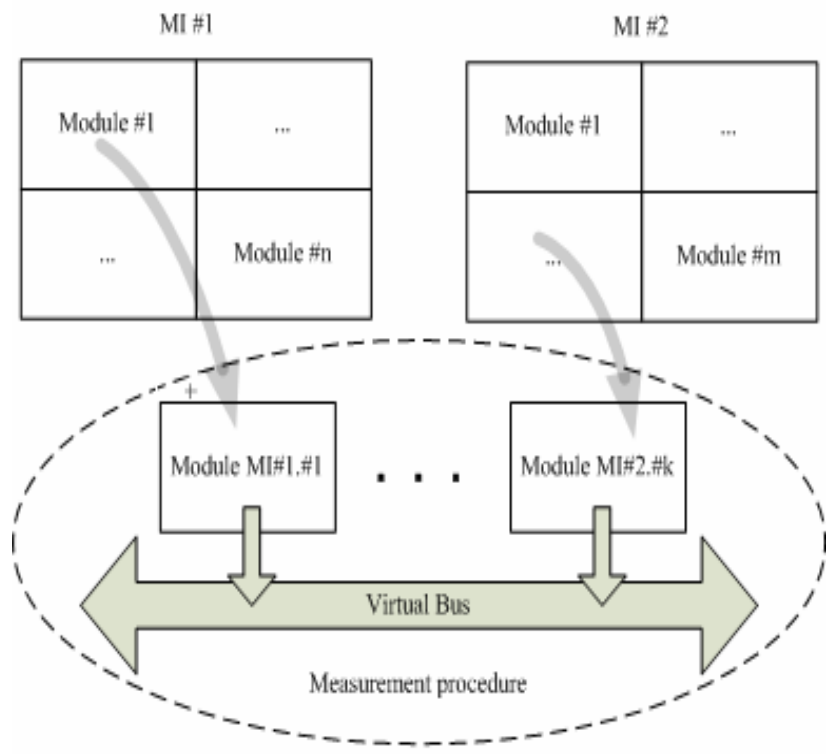

c)

Fig. 1 - Traditional and innovative criteria to build the VI of the measurement procedure.
In the case numerous MIs are employed, the VI implementing the measurement procedure uses the required functionalities of each MI by selecting the corresponding cluster of commands. Also in this case the selected functionalities of the MIs are available always by means of the customized GUI of the measurement procedure.

The implementation of the measurement procedure in only one VI can be advantageous during the design. Indeed, this approach speeds up the realization of the VI. In the contrary, the VI must be changed if new functionalities of the MI need.

The new design approach proposed in [8] is based on splitting up the traditional VI of the MI in several selfcontained modules. The modules are able to exchange data by using the virtual bus in order to execute the measurement procedure.

The criteria to be taken into account and the steps to be followed to define and implement each module are:

step 1. analyze the commands of the MI under consideration and define the different logical functionalities;

step 2. select all the commands to be used to implement each functionality in autonomous and self containing module;

step 3. each module must to be organized to receive the command from the GUI;

step 4. the data are exchanged by the virtual bus, only;

step 5. each module must executes the procedure without exchange of command and data with others modules;

step 6. each module can be written in a different language and communicate with the other module by using specific file;

step 7. each module produce a specific file in a custom XML format in order to save the info and to be easily read by different module written in different language.

In order to highlight the advantages of this approach, Fig. 2 shows the organization of three measurement procedures created on the PDA by splitting the complete traditional VI of the Digital Storage Oscilloscope (DSO) in self containing modules, according to the steps of the design approach. In this case the user downloads on demand by the server only the modules employed in the measurement procedure. Therefore, the occupancy of the memory of the PDA can be reduced to the minimum. Each module can be written in a different language and communicates with another by using the virtual bus, as depicted in Fig.2. The independence among the modules permits to the user the dynamical configuration of the measurement procedure on demand.

The configuration of the measurement procedure is performed on the PDA by organizing the order list of modules by using the specific browser. 


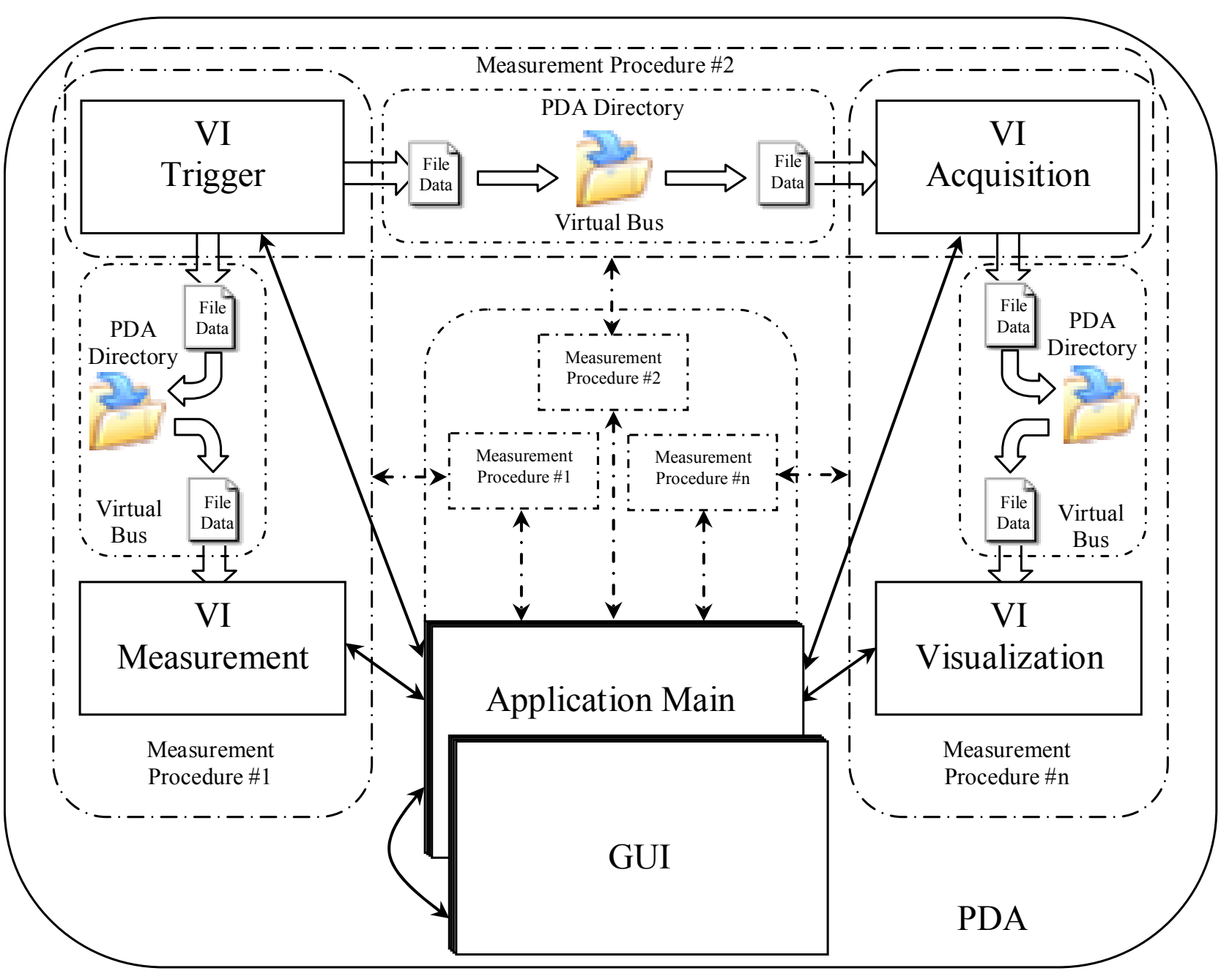

Fig. 2 - Three different measurement procedures realized on PDA by splitting the VI of DSO in self containing modules.

\section{CONCLUSIONS}

The mobile-learning can be considered today the complementary activity to both e-learning and traditional learning. On the basis of this consideration, the research has been addressed to explore the use of the mobile devices in the teaching of the electrical and electronic measurement and instrumentation.

By referring to the delivered services to the students from the Remote Didactic Laboratory Laboratorio Didattico Remoto - LA.DI.RE. "G. Savastano", different solutions have been proposed based on several software architectures and on the characteristics of the mobile devices.

In particular, the functionalities of the mobile devices must integrate with the three asynchronous typologies of the services delivered to the student by the remote measurement laboratory: Experiment Visualization, Experiment Control, and Experiment Creation.

In order to enable the user to view and control the experiment application, the thin client approach was used for visualization of remote experiments on the mobile devices.

In order to enable the user to create the experiment by the mobile device, the new design approach of the innovative VI was used. This approach is based on the organization of the innovative VI, named module, in both autonomous and self containing entities on the basis of the characteristics of the Measurement Instrument.

\section{REFERENCES}

[1] A. Holzinger, A. Nischelwitzer, M. Meisenberger: "Lifelong-Learning Support by M-learning: Example Scenarios", e-Learn magazine, http://www.elearnmag.org/subpage.cfm?section=rese arch\&article $=6-1$.

[2] J. Attewell, C. S. Smith: "Mobile Mobile learning and social inclusion: focusing on learners and learnin", Learning with mobile device,- a book of papers, Learning and Skills Development Agency 2004, www.LSDA.org.uk.

[3] D. Cmuk, M. Borsic, F. Zoino, "Remote versus Classical Laboratory in Electronic Measurements teaching - effectiveness testing", IMEKO XVIII world congress proceedings, Rio de Janeiro 2006.

[4] D. Grimaldi, S. Rapuano, M. Riccio, F Zoino, "Virtual Laboratory as Realistic Tool for the e- 
Learning in the Electric and Electronic Measurement and Instrumentation", International Scientific Journal of Computing, vol. 6, Issue 2, 2007.

[5] Web Services. http://www.w3.org/2002/ws/

[6] D. Grimaldi, S. Rapuano, T. Laopoulos, "Exploring the capability of web-based measurement systems for distance learning", in Distributed Cooperative Laboratories: Networking, instrumentation and measurements, Springer, 2006.

[7] K. Topley: "J2ME in a Nutshell", Publisher: O'Reilly, March 2002, p.9-11.

[8] D. Grimaldi, F. Lamonaca, "Dynamical Configuration of Measurement Procedures on PDA by Using Measurement Application Repository Server", Proc. of IEEE International Workshop on Intelligent Data Acquisition and Advanced Computing Systems: Technology and Applications, 6-8 September 2007, Dortmund, Germany.

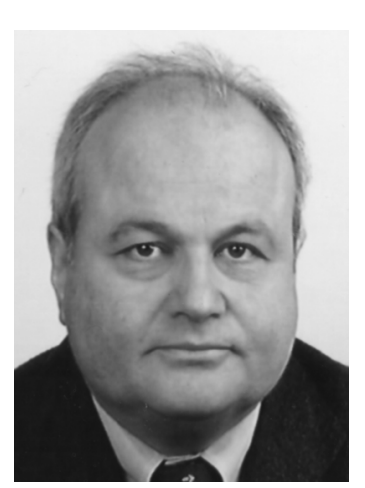

\section{Domenico Grimaldi} received the Dr. Ing. degree "cum laude" in Electrical Engineering from the University of Naples in 1979. $\mathrm{He}$, after working as an independent consultant, in the 1990 joined the Department of Electronic, Computers and System Science (DEIS) of the University of Calabria, Italy, where he is currently Associated Professor of Electronic Measurement. He has remained there in a variety of research and management positions. From 1999 to 2006 he was responsible of the research unit in the frame of National Project PRIN supported by the Italian Ministry for University and Research. From 1997 to 2001 he was responsible in the Tempus Project and Leonardo da Vinci Project supported by the European Union. From 1998 to 2004 he was delegate of the Rector of the University of Calabria. He is a member of the Italian Institute of Electrical Engineers (AEI), and from 1998 to 2000 he was AEI vice-president for the Calabria Region. Since 1994 he is a member of the IEEE. He is coordinator of the Working Group "e-tools for I\&M Education" of the IEEE Instrumentation and Measurement Society, Technical Committee TC-23 "Education in Instrumentation and Measurement".
Prof. Grimaldi has authored and co-authored over 160 papers published in international journals and conference proceedings. His current researches include the characterization of measurement transducers, neural modelling for $A D C$ and measuring systems, digital signal processing for monitoring and testing, virtual instrumentation and distributed measurements, and telecommunication system measurement.

Drago Cmuk is a researcher and assistant at the Faculty of Electrical Engineering and Computing, Zagreb, Croatia and Faculty of Engineering, Benevento, Italy. He graduated 2003. and started international postgraduate studies and scientific work on the field of remote laboratories 2004 mentioned universities.

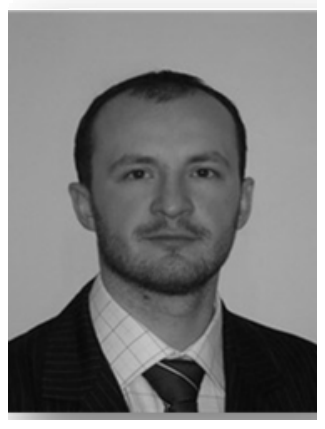

$\mathrm{He}$ is working as assistant on the group of courses on Electric Measurements and Quality management. Currently he is writing dissertation on the topic of optimization of E-learning and m-learning methods in metrology education.

\section{Sergio Rapuano (M'O0)} received the master's degree (cum laude) in electronic engineering Ph.D. degree, from the University of Salerno (Italy), in computer science, telecommunications and applied electromagnetism. Since 2002 he is with the Faculty of Engineering of the University of Sannio as Assistant Professor of Electric

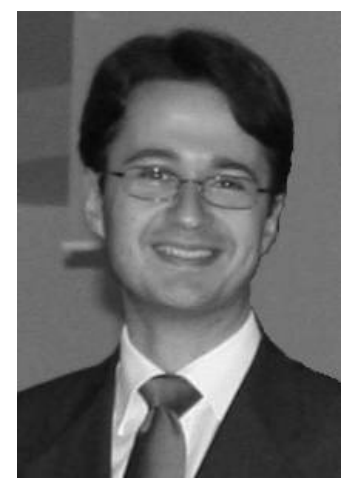
and Electronic Measurement. He is a member of the IEEE Instrumentation and Measurement Society TC-10 and secretary of the TC-23 Working Group on "e-tools for Education in Instrumentation and Measurement". His research interests include digital signal processing for measurement in telecommunications, data converter characterization, distributed measurement systems and virtual laboratories, medical measurement. 